\title{
Structural defects caused by Acrodermatitis Enteropathica mutations in the extracellular domain account for mistrafficking and malfunction of ZIP4
}

\author{
Eziz Kuliyev1, ${ }^{1}$, Chi Zhang ${ }^{2, *}$, Dexin Sui ${ }^{2}$, and Jian $\mathrm{Hu}^{1,2}$ \\ ${ }^{1}$ Department of Chemistry \\ ${ }^{2}$ Department of Biochemistry \& Molecular Biology \\ Michigan State University, East Lansing, MI 48824 \\ ${ }^{*}$ Equally contributive to this work.
}

Corresponding author: Jian Hu, email: hujian1@msu.edu

Running title: Acrodermatitis Enteropathica mutations in ZIP4-ECD

Keywords: Acrodermatitis Enteropathica, ZIP4, zinc transporter, mutation, extracellular domain, mistrafficking, misfolding 


\begin{abstract}
ZIP4 is a representative member of the Zrt-/Irt-like protein (ZIP) transporter family and responsible for zinc uptake from diet. Loss-of-function mutations of human ZIP4 (hZIP4) drastically reduce zinc absorption, causing a life-threatening autosomal recessive disorder, Acrodermatitis Enteropathica (AE). Although the zinc transport machinery is located in the transmembrane domain conserved in the entire ZIP family, half of the missense mutations occur in the extracellular domain (ECD) of hZIP4, which is only present in a fraction of mammalian ZIPs. How the AEcausing mutations in the ECD lead to ZIP4 malfunction has not be fully clarified. In this work, we characterized all the seven confirmed AE-causing missense mutations in hZIP4-ECD and found that the variants exhibited completely abolished zinc transport activity measured in a cell-based transport assay. Although the variants were able to be expressed in HEK293T cells, they failed to traffic to cell surface and were largely retained in the ER with immature glycosylation. When the corresponding mutations were introduced in the ECD of ZIP4 from Pteropus Alecto, a close homolog of hZIP4, the variants exhibited impaired protein folding and reduced thermal stability, which likely account for intracellular mistrafficking of the AE-associated variants and as such a total loss of zinc uptake in cells. This work provides a molecular pathogenic mechanism for AE, which lays out a basis for potential therapy using small molecular chaperones.
\end{abstract}




\section{Introduction}

Zinc is an essential micronutrient for any living organism. As the second most abundant transition metal element in humans after iron, zinc is required for function of over 2,000 transcription factors and activity of approximately 300 enzymes (1). It has been estimated that zinc binds to nearly 3,000 proteins which account for $\sim 10 \%$ of the proteins encoded in human genome (2). Studies have shown that zinc deficiency may affect multiple biological processes, causing growth retardation, immune dysfunction, diarrhea, delayed sexual maturation, and skin lesions (3). Zinc deficiency is usually caused by inadequate zinc supply in foods or acquired diseases either reducing zinc uptake or increasing zinc loss (4). In rare cases, inherited zinc deficiency, which is an autosomal recessive disorder called Acrodermatitis Enteropathica (AE), is caused by loss-offunction (LOF) mutations of ZIP4 $(5,6)$, the exclusive high-affinity zinc transporter in gastrointestinal system responsible for zinc uptake from regular diet (7-10). AE is fatal without treatment, but lifelong high-dose zinc supplementation on daily basis can effectively alleviate the symptoms (11), implying the presence of additional low-affinity zinc absorption mechanism(s).

ZIP4 is a representative member of the Zrt-/lrt-like Protein (ZIP) family (Solute Carrier 39A, SLC39A) consisting of divalent transition metal transporters ubiquitous in all the kingdoms of life (12-18). ZIP4 is specifically expressed on the apical side of enterocytes in small intestine and also in kidney where it is believed to be involved in zinc reabsorption from urine (18). Topologically, ZIP4 has a transmembrane domain (TMD) which is generally conserved in the entire ZIP family and a large extracellular domain (ECD) which is only present in a fraction of the family members $(19,20)$. Be of interest, the AE-causing mutations are evenly distributed along the 12 exons of the zip4 gene without showing hotspot $(21,22)$. As a result, half of the missense AE-causing mutations are mapped in the ECD and the other half in the TMD where the zinc transport machinery is located. A previous work has investigated several corresponding mutations in the TMD of mouse ZIP4 (mZIP4) but only one mutation in the ECD (equivalent to P200L in human ZIP4, hZIP4) was studied in the same report (7). The P200L variant of hZIP4 was characterized in a recent report (23). So far, it is still unclear about how the other AE-causing mutations in the ECD affect hZIP4 function and the corresponding molecular mechanisms.

The crystal structure of the ECD of ZIP4 from Pteropus Alecto (black fruit bat, pZIP4-ECD), which shares $68 \%$ sequence identity with hZIP4-ECD, provides a structural framework to deduce structural impacts of the $A E$-causing mutations on this regulatory domain required for optimal zinc transport (19). In this work, we functionally characterized all the seven confirmed AE-causing 
mutations in a human cell line (HEK293T) and biophysically studied the purified variants of pZIP4ECD harboring the corresponding mutations. We found that all these mutations showed little zinc transport activity in a cell-based transport assay, although their overall expression levels were not much different from that of the wild type protein. The variants exhibited drastically reduced cell surface level and were aberrantly retained in the ER with immature glycosylation. For the purified pZIP4-ECD variants, biophysical studies revealed that the mutations caused reduced a-helical content, undermined packing and decreased thermal stability. Taken together, these findings indicate that impaired protein folding and compromised structural compactness caused by the AE-causing mutations in ZIP4-ECD account for ZIP4 dysfunction linked with intracellular mistrafficking.

\section{Results}

The AE-causing mutations in the ECD of hZIP4 led to total loss of zinc transport activity

A total of 17 missense mutations in hZIP4-ECD have been documented, of which seven are confirmed $\mathrm{AE}$-causing mutations and the others are benign single-nucleotide polymorphisms (SNPs), according to the MASTERMIND database (https://mastermind.genomenon.com/) and an early collection (21). Mapping these residues on the hZIP4-ECD structural model (generated using SWISS-MODEL (24)) shows that the residues subjected to AE-causing mutations (C62R, R95C, A99T, N106K, P200L, Q303H and C309Y) are all in the structured regions, whereas most of the residues where the SNPs occur are on loops or in disordered regions (Figure 1). In this work, we characterized all the seven AE-causing mutations, including four homozygous mutations (C62R, P200L, Q303H and C309Y) (5,6,25,26) and three heterozygous mutations (R95C, A99T and N106K) $(6,26,27)$, and studied their impacts on hZIP4 function. The hZIP4 variants with a Cterminal HA tag were transiently expressed in HEK293T cells and applied to the cell-based radioactive zinc transport assay $(6,7,28,29)$. As shown in Figure 2A, in sharp contrast to the wild type hZIP4, none of the cells expressing any variants uptook more zinc than the blank group in which the cells were transfected with an empty vector, which means that the variants have no detectable zinc transport activity with $10 \mu \mathrm{M}$ of $\mathrm{Zn}^{2+}$ added in culture media. For the wild type hZIP4, the previous studies have shown that zinc transport activity reaches plateau at $10 \mu \mathrm{M}$ of $\mathrm{Zn}^{2+}$ under the same condition $(28,29)$. To examine whether any activity can be detected at higher zinc concentration, the $\mathrm{P} 200 \mathrm{~L}$ variant was tested at various zinc concentrations up to $50 \mu \mathrm{M}$, but the results only confirmed no detectable activity (Figure S1). 
The AE-associated variants exhibited immature glycosylation

The previous study on mZIP4 has shown that the AE-causing mutations in the TMD led to significantly lowered expression levels, likely due to accelerated degradation (7). To examine whether the AE-causing mutations in the ECD have similar effects, the expression levels of the variants were detected in the whole cell samples using Western blots with an anti-HA antibody. As shown in Figure 2B, the overall expression levels of the variants are comparable to or modestly lower than that of the wild type hZIP4. However, the wild type hZIP4 has two discrete bands at approximately $75 \mathrm{kDa}$, whereas the $\mathrm{AE}$-associated variants have only one corresponding to the lower band of the wild type protein. It has been determined that the upper band represents the maturely glycosylated form whereas the protein in the lower band is immaturely glycosylated (7), which is further confirmed in this work using PNGase $F$, an enzyme cleaving $N$-linked glycoside bond(s) (Figure S2). Thus, the total expression of any variants is not affected by the mutations in the ECD, but a defect in glycosylation was observed for all the AEassociated variants.

\section{$N$-glycosylation is not required for hZIP4 activity}

Proteins expressed at cell surface are often modified by $N$-glycosylation, which may play a key role in protein function (30). As the AE-associated variants losing zinc transport activity is concomitant with glycosylation defects, we asked whether the lack in glycosylation is responsible for activity loss. Using the NetNGlyc server (http://www.cbs.dtu.dk/services/NetNGlyc/), we identified a potential glycosylation site at N261 in an "NxS/T" motif. We then generated the N261Q variant and expressed it in HEK293T cells. As expected, the N261Q variant showed only the lower band in Western blot (Figure 2B), confirming N261 is indeed the only N-glycosylation site in hZIP4. Importantly, zinc transport assay of the N261Q variant only showed a modest reduction of transport activity (Figure 2C), indicating that glycosylation at N261 is not pivotal for zinc transport and therefore lack of glycosylation of the $A E$-associated variants does not account for loss of zinc transport activity.

\section{The AE-associated variants have significantly diminished cell surface level}

As the glycan are added to the client membrane proteins in the ER and processed in the ER and then in the Golgi in a stepwise manner (31), immature glycosylation is an indicator of defect in intracellular trafficking. To examine potential mislocalization, we tested cell surface expression levels of the AE-associated variants by applying the anti-HA antibody to the non-permeabilized 
cells fixed with formaldehyde. After extensive wash, non-specific bound antibody was removed, leaving those specifically bound with the C-terminal HA tag at cell surface. As shown in Figure 3A, all the AE-associated variants had substantially reduced cell surface levels when compared to the wild type hZIP4 in Western blots. Consistent with the zinc transport data, the N261Q variant missing the $N$-linked glycan had significantly higher level of cell surface expression than the variants linked with disease, indicating that the $N$-glycosylation at N261 is not a key factor for hZIP4 surface expression. We then applied immunofluorescence imaging to locate the AEassociated variants expressed in cells. To do that, the cells transiently expressing the wild type or the variants were fixed and permeabilized with formaldehyde and TX-100, followed by staining with an FITC-labeled anti-HA antibody. The samples were then checked under confocal laser scanning microscope (CLSM). Consistent with the Western blot results shown in Figure 2B, the wild type and the variants were all expressed in HEK293T cells at comparable levels with most of the staining signals within the cytoplasm (Figure 3B). To detect the transporters expressed at cell surface, the cells were fixed with formaldehyde and treated with the same anti-HA antibody, followed by extensive washing and checking under CLSM. In Figure 3B, fluorescence signals at cell surface can be convincingly detected for the wild type hZIP4 and the N261Q variant, whereas the signals of the AE-associated variants imaged under the same conditions were much weaker or not detectable, which is consistent with the Western blot data shown in Figure 3A. Collectively, our data strongly indicate that the cell surface expression levels of the AE-associated variants are largely suppressed, which is likely responsible for loss of zinc transport activity.

\section{The AE-associated variants are retained in the $E R$}

Since all the AE-associated variants were expressed but not presented at cell surface, we then asked where the variants are located in cells. To determine intracellular location of the variants, we examined co-localization of hZIP4 and its variants with an ER-resident protein, calreticulin. As shown in Figure 4, the staining of the wild type hZIP4-HA (red) is partially overlapped with those of calreticulin (green), resulting in a yellow/orange color for the overlapped portion. This result suggests that a portion of hZIP4 in HEK293T cells are in the ER, at least partially due to overexpression driven by the strong CMV promoter in transient transfection. However, as significant amount of hZIP4 is not co-localized with calreticulin, this portion of the wild type hZIP4 is either in other organelles (such as the Golgi complex) or at the plasma membrane. In contrast, the AE-associated variants are mostly co-localized with calreticulin, strongly suggesting that the variants are primarily retained in the ER. As membrane proteins are initially glycosylated in the $\mathrm{ER}$ and then the glycans are further processed in the Golgi complex for maturation, the variants 
being retained in the ER is consistent with immature glycosylation of the variants. It is known that mistrafficking can be the result of protein misfolding and/or structural alteration imposed by disease-causing mutations, we are wondering whether and how the AE-causing mutations affect protein structure. Given the difficulty of obtaining adequate amount of purified full length hZIP4 or hZIP4-ECD to conduct biophysical characterization, we turned to the isolated pZIP4-ECD, which we have previously crystallized and biochemically characterized $(19,28)$. Given the high sequence identity, we believe that the results obtained on purified pZIP4-ECD would help to understand how the disease-causing mutations affect the biophysical properties of the human counterpart.

\section{The AE-causing mutations alter secondary structure of $p Z I P 4-E C D$}

We introduced mutations in pZIP4-ECD on the residues topologically equivalent to those in hZIP4 (C64R, R87C, A91T, D98K, P193L, Q299H and C305Y) and expressed the variants in the same way as for the wild type protein. We have noticed that, even for the wild type pZIP4-ECD, a large portion of purified protein from the Co-affinity column are not well folded as indicated in ionexchange chromatography experiment, which is likely due to the presence of eight invariant cysteine residues required for four disulfide bonds formation. As a result, only a small fraction of expressed protein can be purified to homogeneity for later structural and biophysical experiments, making the overall yield of the wild type pZIP4-ECD barely higher than $1 \mathrm{mg}$ per liter culture. For the variants, we found the misfolding issue is more severe, which led to further lower yield for most of the variants (0.1-0.5 mg/L). Particularly, the yields of two variants (D98K and C305Y) were too low to allow for further characterization. Therefore, we focused on the other five variants in later study.

During purification, we noticed that all the variants were eluted in size-exclusion chromatography with an apparent molecular weight of approximately $60 \mathrm{kDa}$, indicative of a homodimer as the wild type protein, so the AE-causing mutations do not affect ECD dimerization. The purified proteins were applied to circular dichroism (CD) spectrometer and the spectral data were analyzed on the K2D3 server to estimate secondary structure contents (32). As shown in Figure 5, the CD spectrum of the wild type pZIP4-ECD is of high a-helical content (estimated to be 66\%) with the characteristic minima at $208 \mathrm{~nm}$ and $222 \mathrm{~nm}$ and maximum at $195 \mathrm{~nm}$, which is consistent with the reported crystal structure (19). When compared to the wild type protein, all the variants have lower levels of helical content - C64R (61\%), R87C (55\%), A91T (58\%), P193L (64\%) and Q299H $(56 \%)$. We also noticed the ratios of ellipticities $(\theta)$ at $222 \mathrm{~nm}$ and $208 \mathrm{~nm}$ were reduced for all the variants. The $\theta_{222} / \theta_{208}$ ratio is an indicator of whether the helices are packed to form coiled- 
coil like tertiary structures $(33,34)$. When the value is smaller than 1 , it may indicate poorly packed helices and lack of coiled-coils. For the wild type protein $\theta_{222} / \theta_{208}$ is 1.08 , which is consistent with the crystal structure where two helix bundles form two separated subdomains: in the N-terminal helix-rich domain (HRD), a4 is surrounded and packed with the other eight helices, whereas four helices $(\alpha 10-\alpha 12)$ in the C-terminal domain (PAL motif-containing domain, PCD) are packed against with the counterparts from the other monomer to form a homodimer (19). For all the tested variants, we found the $\theta_{222} / \theta_{208}$ ratios were reduced to be smaller than one - C64R (0.93), R87C (0.83), A91T (0.93), P193L (0.98) and Q299H (0.84). Therefore, lower helical content and reduced $\theta_{222} / \theta_{208}$ ratio revealed by the CD spectra indicate defects in protein folding for all the variants, of which R87C and Q299H have greatest effects whereas P193L has the least.

The AE-causing mutations affect thermal stability and heat denaturation-associated thermodynamic parameters of pZIP4-ECD

Next, we compared the thermal stabilities of the wild type and the variants of pZIP4-ECD by gradually increasing temperature and recording changes of ellipticity at $222 \mathrm{~nm}$ in CD spectrum as an indicator of folding status. For the wild type protein, increasing temperature from $20^{\circ} \mathrm{C}$ to $95^{\circ} \mathrm{C}$ was accompanied with a decrease of $\theta_{222}$ and the plateau was reached at the end (Figure 6) when the protein was mostly denatured with a-helical content dropping from $66 \%$ to $20 \%$ (Figure S3). Although the ECD has two structurally distinct domains (HRD and PCD) with the PCD dimerizing through a large hydrophobic interface, the presence of only one transition in the heat denaturation curve suggests that the physical interactions between two domains may synchronize their unfolding processes. We also noticed that, when the heat-denatured protein was allowed to refold at room temperature, majority of the refolded protein is still a homodimer in solution as indicated in size exclusion chromatography, even though the a-helical content is decreased by $10 \%$ and the $\theta_{222} / \theta_{208}$ ratio is reduced to 0.82 (Figure S3). This result suggests that dimerization is either preserved at $95{ }^{\circ} \mathrm{C}$ or re-formed during protein refolding. In either scenario (or a mixed process), this result indicates that pZIP4-ECD has a strong tendency to keep and/or form a dimer, which is consistent with the proposed ECD function of facilitating dimerization of ZIP4 (19). By plotting normalized $\Delta \theta_{222}$ against temperature, the melting temperature $\left(T_{m}\right)$, at which the unfolding process reaches $50 \%$ during heat denaturation, can be estimated. When compared to the $T_{m}$ of the wild type protein $\left(59.5^{\circ} \mathrm{C}\right)$, all the variants except for $\mathrm{Q} 299 \mathrm{H}$ exhibited lowered $\mathrm{T}_{\mathrm{mS}}$, ranging from $56.2^{\circ} \mathrm{C}$ to $58.1^{\circ} \mathrm{C}$ (Figure 6). The unexpected high $\mathrm{T}_{\mathrm{m}}$ for the Q299H variant $\left(61.4^{\circ} \mathrm{C}\right)$ is accompanied with a more flattened heat denaturation curve when compared 
to the sigmoid curves of the wild type or the other variants, which is implicative of impaired cooperativity and interaction network of the structural elements of this variant.

Taken together, the CD spectra and the heat denaturation experiments of pZIP4-ECD variants revealed substantially changed biophysical properties, indicating that the substitutions in pZIP4$E C D$, which are equivalent to the AE-causing mutations in hZIP4-ECD, lead to impaired protein folding and decreased thermal stability.

\section{Discussion}

As the ZIP family plays a central role in transition metal homeostasis and cell signaling (18), LOF mutations of human ZIPs cause severe syndromes, including AE which is the first genetic disorder discovered to be associated with the ZIP family $(5,6)$. So far, at least seven AE-causing mutations have been reported and some of them have been biochemically studied on mZIP4, a close homolog of hZIP4. Most of the missense mutations in the TMD of mZIP4 led to lower expression level, reduced cell surface expression and largely diminished zinc transport activity (7). Mapping the mutations on the structural model of hZIP4 generated using a bacterial ZIP structure has suggested that the involved residues appear to play structural roles and therefore the AE-causing mutations in the TMD likely affect protein folding and/or stability (35), which would eventually lead to degradation by quality control mechanism in the ER (31). The only characterized mutation in the ECD of mZIP4 is P200L, which is equivalent to P200L in hZIP4 (7). The same mutation in hZIP4 was also reported in a recent study (23). In this work, we characterized seven confirmed AE-causing mutations in the ECD (including P200L) and found that all the variants exhibited similar behavior when expressed in HEK293T cells - they were able to be expressed at similar (or modestly reduced) levels as the wild type hZIP4 but barely detected at cell surface, which accounts for the total loss of zinc transport activity. The fact that the variants are immaturely glycosylated and aberrantly trapped in the ER strongly indicates intracellular mistrafficking. By biophysically examining the effects of the corresponding mutations in pZIP4-ECD, we demonstrated that the mutations cause defects in folding and stability, which provides a causal explanation for mistrafficking and dysfunction of the AE-associated hZIP4 variants.

Protein misfolding and structural defects are known pathogenic mechanisms for dysfunction of membrane proteins caused by disease-linked mutations. A well-documented case is the autosomal recessive disorder cystic fibrosis (CF) which is caused LOF mutations of a chloride ion channel, cystic fibrosis transmembrane conductance regulator (CFTR). Notably, the most 
prevalent mutation ( $>90 \%$ ) associated with CFTR is $\triangle F 508$, for which the residue F508 within the cytosolic nucleotide binding domain 1 (NBD1) is missed (36). The $\Delta$ F508 variant exhibited drastically reduced transport activity, immature glycosylation, reduced surface expression and accelerated clearance from plasma membrane (37). The atomic resolution of cryo-EM structures of CFTR have shown that F508 is buried in a hydrophobic core $(38,39)$ which mediates physical interactions of the NBD1 and the TMD, and biochemical/biophysical studies have indicated that the variant has defects in folding and structural compactness (37). Remarkably, the AE-linked variants with single residue substituted in the ECD showed a similar phenotype as the $\triangle F 508$ CFTR variant. As discussed in our previous report and also shown in Figure 1, the AE-causing mutations occur at the conserved residues in structured regions where the involved residues play structural roles in formation of disulfide bonds (C62R, C309Y), salt bridge (R95C), hydrogen bonds (N106K and Q303H), and hydrophobic cores (A99T, P200L). With no surprise, the biophysical studies on pZIP4-ECD indicated that the tested five variants (C64R, R87C, A91T, P193L, and Q299H) have shown defects in folding and structural integrity, which are manifested by reduced $\alpha$-helical content and the $\theta_{222} / \theta_{208}$ ratio (for all the variants), and decreased thermal stability (with the exception of the Q299H variant) (Figures 5 and 6). The extremely low yield for the D98K and C305Y variants already implies severe defects in folding and/or stability.

We notice the discrepancy between our results and other reports on the P200L variant. It was shown that the P200L variant of mZIP4 was expressed in HEK293T cells with a cell surface level comparable to that of the wild type protein and a $70 \%$ decrease in zinc transport activity (7). In contrast, the P200L variant of hZIP4 showed little surface expression and no detectable activity in this work (Figures 2 \& 3). As already mentioned in the mZIP4 study, nearly all the wild type mZIP4 was maturely glycosylated, whereas only $50 \%-70 \%$ of the P200L variant did so, suggesting that a significant amount of the variant was not properly processed. Notably, even for the wild type hZIP4, the percentage of maturely glycosylated protein is just about $50 \%$ (Figures 2 and $\mathbf{3}$ ). It is unclear why mZIP4 is more efficiently processed and traffic in a human cell line than hZIP4. One possibility would be that mZIP4 is intrinsically more stable than hZIP4 so that it has better chance to fold correctly in the ER. It would also explain why the P200L variant of mZIP4 can still be presented at cell surface despite of folding defect indicated by immature glycosylation. While this work was being conducted, we noticed a study reporting that the P200L variant of hZIP4 expressed in HEK293T cells was shown to have a normal zinc transport activity which was measured by using a small molecule zinc-responsive fluorescence dye (23). It is unclear whether this discrepancy is due to different approaches used for tracing zinc transport. Radioactive ${ }^{65} \mathrm{Zn}$ 
based transport assay has been used to study transport kinetics of a variety of ZIPs, including hZIP4 (7,19,28,29,35,40-44). Consistent with loss of zinc transport activity, the P200L variant of hZIP4 is shown to be barely expressed at cell surface. We detected cell surface expression levels of hZIP4 and its variants using two approaches following the previous mZIP4 study (7) - Western blot for detecting cell surface bound anti-HA antibody and immunofluorescence imaging for visualizing fluorescence-labeled anti-HA antibody associated at cell surface. As shown in Figures

$3 \mathrm{~A}$ and $3 \mathrm{~B}$, the results of both approaches consistently indicated that the $A E$-associated variants, including the P200L variant, had much lower levels of cell surface expression than the wild type hZIP4. Co-localization experiment further revealed that the P200L variant is largely retained in the ER (Figure 4), which explains the lack of zinc transport activity of this variant.

After the discovery of the causal link between ZIP4 mutations and AE, disease-causing mutations have been reported in other human ZIPs. The LOF mutations of ZIP13 cause the spondylocheirodysplastic form of Ehlers-Danlos syndrome (SCD-EDS) $(42,45,46)$, an autosomal recessive disorder characteristic of defects of connective tissues, bones, and teeth. It has been demonstrated that both the missense mutation G64D and the deletion mutation $\triangle F L A$ (on the third transmembrane helix) drastically reduced stability and protein expression level in cells due to accelerated clearance through the ubiquitination dependent proteasome degradation pathway (45). Recently, inherited LOF mutations of ZIP8 and ZIP14, which are close homologs with broad substrate spectrum covering from the beneficial trace elements (zinc, iron, manganese) to the toxic cadmium, have been linked with severe failure of systemic manganese homeostasis $(47,48)$. The disease-linked ZIP8 variants fail to transport $\mathrm{Mn}^{2+}$ because of abrogated cell surface expression and being aberrantly retained in the ER (49). Notably, these ZIP8 variants harbor at least one substitution in the ECD, highlighting the importance of the ECD in ZIP8 intracellular trafficking. In this work and also in our previous report, we demonstrate that the mutations on the conserved residues in the ECD significantly affected ZIP4 intracellular trafficking (19). Although the exact role of the ECD is still not fully clarified, these studies have suggested that correct folding and structural integrity of the ECD are required for proper intracellular trafficking of the ZIPs. Whether the ECD plays any active roles in folding, processing or trafficking of mammalian ZIPs would be interesting to investigate later.

In summary, seven confirmed AE-causing mutations were functionally and biophysically characterized in this work, and the results indicate that the mutations in the ECD lead to protein mistrafficking because of impaired folding, reduced thermal stability and undermined structural integrity, which accounts for the abolished zinc transport activity. Given that AE and CF share a 
similar molecular pathogenic mechanism and that small molecule chemical chaperones have been successfully applied for CF treatment (50), developing AE-specific chemical chaperones may represent an alternative strategy to treat this rare disease. After all, life-time and high-dose zinc administration may cause imbalance of other trace elements, such as copper deficiency $(51,52)$.

\section{Experimental procedures}

Genes, plasmids and mutagenesis

The plasmids harboring hZIP4 (GenBank code: BC062625) or pZIP4-ECD (gene ID: ELK11751, residue 36-322) are the same as reported (19). All the site-directed mutations were conducted using QuikChange mutagenesis kit (Agilent, Cat\# 600250) and verified by DNA sequencing.

\section{Cell culture, transfection and Western blot}

Human embryonic kidney cells (HEK293T, ATCC, Cat \#CRL-3216) were cultured in Dulbecco's modified eagle medium (DMEM, Thermo Fisher Scientific, Invitrogen, Cat\#11965092) supplemented with $10 \%(\mathrm{v} / \mathrm{v})$ fetal bovine serum (FBS, Thermo Fisher Scientific, Invitrogen, Cat\#10082147) and Antibiotic-Antimycotic solution (Thermo Fisher Scientific, Invitrogen, Cat\# 15240062) at $5 \% \mathrm{CO}_{2}$ and $37^{\circ} \mathrm{C}$. Cells were seeded on poly-D-lysine (Corning, Cat\# 354210) coated 24-wells trays for 16 hours in the basal medium and transfected with 0.5-0.8 $\mu \mathrm{g}$ DNA/well by lipofectamine 2000 (Thermo Fisher Scientific, Invitrogen, Cat\# 11668019) in OPTI-MEM medium.

For Western blot, all the samples were heated at $96{ }^{\circ} \mathrm{C}$ for $6 \mathrm{~min}$ before loading on SDS-PAGE gel. The protein bands were transferred to PVDF membranes (Millipore, Cat\# PVH00010). After being blocked with $5 \%$ non-fat dry milk, the membranes were incubated with anti-HA antibody (Thermo Fisher Scientific, Cat\#26183) at $4{ }^{\circ} \mathrm{C}$ overnight. Bound primary antibodies were detected with HRP-conjugated goat anti-mouse immunoglobulin-G at 1:5,000 (Cell Signaling Technoloy, Cat\# 7076S) or goat anti-rabbit immunoglobulin-G at 1:2,500 (Cell Signaling Technology, Cat\# 7074S) by chemiluminescence (VWR, Cat\# RPN2232). The blots were taken using a Bio-Rad ChemiDoc ${ }^{\mathrm{TM}}$ Imaging System.

\section{Zinc transport assay}

Twenty hours post-transfection, cells were washed with the wash buffer (10 mM HEPES, $142 \mathrm{mM}$ $\mathrm{NaCl}, 5 \mathrm{mM} \mathrm{KCl}, 10 \mathrm{mM}$ glucose, $\mathrm{pH}$ 7.3) followed by incubation with $10 \mu \mathrm{M} \mathrm{ZnCl}$ (containing $30 \%{ }^{65} \mathrm{ZnCl}_{2}$ ) in Chelex-treated $10 \% \mathrm{FBS} / \mathrm{DMEM}$ media for 20 mins at $37^{\circ} \mathrm{C}$. Then, plates were 
transferred on ice and zinc uptake was stopped by addition of precooled 1 mM EDTA containing wash buffer. Cells were centrifuged at $3500 \mathrm{rpm}$ and the supernatant was discarded. Cells were washed twice before lysis with 0.5\% Triton X-100. Packard Cobra Auto-Gamma counter was used to detect radioactivity. The same procedure was used for activity assay of the P200L variant at designated zinc concentrations. The cells transfected with the empty vector were treated in the same way.

\section{hZIP4-HA surface expression detection}

hZIP4-HA expressed at the plasma membrane was indicated by the surface bound anti-HA antibodies recognizing the $\mathrm{C}$-terminal HA tag of hZIP4. After $24 \mathrm{~h}$ transfection, cells were washed twice with DPBS on ice and then fixed for $10 \mathrm{~min}$ in $4 \%$ formaldehyde at room temperature. Cells were then washed three times in DPBS (5 minutes each wash) and incubated with $2.5 \mu \mathrm{g} / \mathrm{ml}$ antiHA antibody diluted with $5 \%$ BSA in DPBS for $1.5 \mathrm{~h}$ at room temperature. Cells were washed seven times with DPBS to remove unbound antibodies and then lysed in SDS-PAGE loading buffer. The anti-HA antibody bound to the surface hZIP4-HA in cell lysates were detected in Western blot with HRP-conjugated goat anti-mouse immunoglobulin-G (1:5000). As loading control, $\beta$-actin levels were detected using an anti- $\beta$-actin antibody $(1: 2,500)$.

\section{Immunofluorescence imaging}

HEK293T cells were grown in 24-well trays for $16 \mathrm{~h}$ on sterile glass coverslips and transfected with plasmids harboring the genes of hZIP4 or its variants using lipofectamine 2000 . To visualize cell surface expressed hZIP4 or its variants, cells were washed briefly by DPBS after $24 \mathrm{~h}$ transfection and then fixed for $10 \mathrm{~min}$ at room temperature using $4 \%$ formaldehyde. The cells were washed in DPBS (5 minutes each wash) and incubated with $2.5 \mu \mathrm{g} / \mathrm{ml}$ an FITC-labeled antiHA antibody (Sigma, Product \# H7411) diluted with 5\% BSA in DPBS for $1.5 \mathrm{~h}$ at room temperature. After five washes with DPBS, coverslips were mounted on slides with fluoroshield mounting medium with DAPI (Abcam, Cat\# ab104139). Images were taken with a 40X objective using a Spectral-based Olympus FluoView 1000 confocal laser scanning microscope (CLSM). To detect intracellular localization of hZIP4 variants and calreticulin (an ER marker), after fixation by using formaldehyde, cells were permeabilized and blocked for $1 \mathrm{~h}$ with DPBS containing $5 \%$ goat serum (Cell Signaling Technology, Cat\# 5425S) and 0.1\% Triton X-100 and then incubated with anti-HA antibodies at 1:500 (Thermo Fisher Scientific, Cat\#26183) and/or anti-calreticulin antibodies at 1:300 (Thermo Fisher Scientific, Cat\#PA3-900) at $4{ }^{\circ} \mathrm{C}$ for overnight. After three washes with DPBS (5-minute incubation for each wash), cells were incubated with Alexa-568 goat 
anti-mouse antibodies at 1:500 (Thermo Fisher Scientific, Cat\# A-11004) and Alexa-488 antirabbit antibodies at 1:500 (Thermo Fisher Scientific, Cat\# A-27034). After 3 washes with DPBS, coverslips were mounted on slides with fluoroshield mounting medium with DAPI (Abcam, Cat\# ab104139). Images were taken with a 100X objective.

\section{Expression and purification of pZIP4-ECD and the variants}

The wild type pZIP4-ECD and the variants were expressed as previously described (19). In brief, the E.coli cells of Origami ${ }^{\mathrm{TM}} \mathrm{B}(\mathrm{DE} 3)$ pLysS (Novagen) transformed with the pLW01 vector were grown at $37{ }^{\circ} \mathrm{C}$ in lysogeny broth medium until $\mathrm{OD}_{600} \sim 0.6$, and expression was induced by 0.2 mM IPTG before the cells were transferred to $16{ }^{\circ} \mathrm{C}$ for overnight growth. After cell lysis by sonication, the His-tagged proteins were purified using a nickel-nitrilotriacetic acid column. After removal of $\mathrm{N}$-terminal $\mathrm{His}_{6}$-tag by thrombin, the proteins were dialyzed against a Tris-HCl buffer (pH 8.0) containing $10 \mathrm{mM}$ EDTA, subjected to ion-exchange chromatography (Mono-Q, GE Healthcare), and then polished by size-exclusion chromatography (Superdex 200 10/300 GL, GE Healthcare) in a buffer containing 10 mM HEPES, pH 7.3 and $100 \mathrm{mM} \mathrm{NaCl}$.

\section{Circular dichroism experiments}

To prepare the samples for CD experiments, Thermo Scientific Slide-A-Lyzer MINI Dialysis Device was used for changing the sample buffer to $10 \mathrm{mM}$ phosphate $\mathrm{pH} 7.3$ over the course of a two-day dialysis at $4{ }^{\circ} \mathrm{C}$ with buffer change for three times. Sample concentrations were measured by using a NanoDrop ND-1000 Spectrophotometer and then adjusted to proper concentration using the dialysis buffer.

The circular dichroism (CD) spectrum of $8 \mu \mathrm{M}$ pZIP4-ECD (or the variants) in $300 \mu \mathrm{l}$ of $10 \mathrm{mM}$ phosphate buffer $(\mathrm{pH} 7.3)$ were recorded in Hellma® $1 \mathrm{~mm}$ QS cuvette sealed with PTFE stopper by using a JASCO-815 CD Spectrometer. Wavelength range was set between 190-260 nm, and data was collected at $1 \mathrm{~nm}$ wavelength increments with $1.5 \mathrm{~s}$ averaging time per wavelength point. Three scans were recorded and averaged. The K2D3 server (http://cbdm-01.zdv.unimainz.de/ andrade/k2d3/n) was used to estimate secondary structure components of the proteins. For thermal stability analysis of wild type protein, $C D$ spectra were recorded in the range of $195-260 \mathrm{~nm}$ and starting temperature was set at $20^{\circ} \mathrm{C}$. Temperature was increased in $10{ }^{\circ} \mathrm{C}$ increments and waiting time was set to 5 minutes at each designated temperature before scanning. Three scans were recorded and averaged. After reaching $95{ }^{\circ} \mathrm{C}$, temperature was cooled down to $20^{\circ} \mathrm{C}$ and waited for 10 minutes before scanning. Three scans were recorded and averaged. For thermal stability study, ellipticities at $222 \mathrm{~nm}$ were recorded while the 
temperature increased from $20^{\circ} \mathrm{C}$ to $95^{\circ} \mathrm{C}$. Rate of temperature increase was $3{ }^{\circ} \mathrm{C} / \mathrm{min}$ with a temperature tolerance of $0.15^{\circ} \mathrm{C}$. The average of three repeats was used in data processing and analysis. Unfolded fraction at temperature $T\left(F_{\text {unfolded, } T}\right)$ was calculated as below:

$F_{\text {unfolded,T }}=\left|\theta_{222,20}-\theta_{222, T}\right| /\left|\theta_{222,20}-\theta_{222,95}\right|$

where $\theta_{222,20}, \theta_{222,95}$ and $\theta_{222, T}$ are the readings of $\theta_{222}$ at $20{ }^{\circ} \mathrm{C}, 95{ }^{\circ} \mathrm{C}$ and temperature $\mathrm{T}$, respectively. Tm was determined from the heat denaturation curve at which $\mathrm{F}_{\text {unfolded }}=0.5$. 


\section{Acknowledgments}

We thank Dr. Jin He at Department of Biochemistry and Molecular Biology for access to fluorescence microscopy for initial sample inspection. We thank Dr. Melinda Frame at Center for Advanced Microscopy for providing assistance in Confocal experiments.

\section{Author contributions}

J.H. conceived the project and designed the experiments; E.K., C.Z. and D.S. conducted experiments; E.K., C.Z. and J.H. analyzed the data and wrote the manuscript.

\section{Funding}

This work is supported by NIH R01GM115373 (to J.H.).

The content is solely the responsibility of the authors and does not necessarily represent the official views of the National Institutes of Health.

\section{Conflict of interest}

The authors declare that they have no conflicts of interest with the contents of this article. 


\section{References}

1. McCall, K. A., Huang, C., and Fierke, C. A. (2000) Function and mechanism of zinc metalloenzymes. J Nutr 130, 1437S-1446S

2. Andreini, C., Banci, L., Bertini, I., and Rosato, A. (2006) Counting the zinc-proteins encoded in the human genome. J Proteome Res 5, 196-201

3. Maret, W., and Sandstead, H. H. (2006) Zinc requirements and the risks and benefits of zinc supplementation. J Trace Elem Med Biol 20, 3-18

4. $\quad$ Prasad, A. S. (2003) Zinc deficiency. BMJ 326, 409-410

5. Kury, S., Dreno, B., Bezieau, S., Giraudet, S., Kharfi, M., Kamoun, R., and Moisan, J. P. (2002) Identification of SLC39A4, a gene involved in acrodermatitis enteropathica. Nat Genet 31, 239-240

6. Wang, K., Zhou, B., Kuo, Y. M., Zemansky, J., and Gitschier, J. (2002) A novel member of a zinc transporter family is defective in acrodermatitis enteropathica. Am J Hum Genet 71, 66-73

7. Wang, F., Kim, B. E., Dufner-Beattie, J., Petris, M. J., Andrews, G., and Eide, D. J. (2004) Acrodermatitis enteropathica mutations affect transport activity, localization and zincresponsive trafficking of the mouse ZIP4 zinc transporter. Hum Mol Genet 13, 563-571

8. Dufner-Beattie, J., Wang, F., Kuo, Y. M., Gitschier, J., Eide, D., and Andrews, G. K. (2003) The acrodermatitis enteropathica gene ZIP4 encodes a tissue-specific, zinc-regulated zinc transporter in mice. J Biol Chem 278, 33474-33481

9. Dufner-Beattie, J., Weaver, B. P., Geiser, J., Bilgen, M., Larson, M., Xu, W., and Andrews, G. K. (2007) The mouse acrodermatitis enteropathica gene Slc39a4 (Zip4) is essential for early development and heterozygosity causes hypersensitivity to zinc deficiency. Hum Mol Genet 16, 1391-1399

10. Geiser, J., Venken, K. J., De Lisle, R. C., and Andrews, G. K. (2012) A mouse model of acrodermatitis enteropathica: loss of intestine zinc transporter ZIP4 (Slc39a4) disrupts the stem cell niche and intestine integrity. PLoS Genet 8, e1002766

11. Perafan-Riveros, C., Franca, L. F., Alves, A. C., and Sanches, J. A., Jr. (2002) Acrodermatitis enteropathica: case report and review of the literature. Pediatr Dermatol 19, 426-431

12. Kambe, T., Yamaguchi-Iwai, Y., Sasaki, R., and Nagao, M. (2004) Overview of mammalian zinc transporters. Cell Mol Life Sci 61, 49-68

13. Liuzzi, J. P., and Cousins, R. J. (2004) Mammalian zinc transporters. Annu Rev Nutr 24, 151172

14. Lichten, L. A., and Cousins, R. J. (2009) Mammalian zinc transporters: nutritional and physiologic regulation. Annu Rev Nutr 29, 153-176

15. Kambe, T., Hashimoto, A., and Fujimoto, S. (2014) Current understanding of ZIP and ZnT zinc transporters in human health and diseases. Cell Mol Life Sci 71, 3281-3295

16. Kambe, T., Tsuji, T., Hashimoto, A., and Itsumura, N. (2015) The Physiological, Biochemical, and Molecular Roles of Zinc Transporters in Zinc Homeostasis and Metabolism. Physiol Rev 95, 749-784

17. Hara, T., Takeda, T. A., Takagishi, T., Fukue, K., Kambe, T., and Fukada, T. (2017) Physiological roles of zinc transporters: molecular and genetic importance in zinc homeostasis. J Physiol Sci 67, 283-301 
18. Jeong, J., and Eide, D. J. (2013) The SLC39 family of zinc transporters. Mol Aspects Med 34, 612-619

19. Zhang, T., Sui, D., and Hu, J. (2016) Structural insights of ZIP4 extracellular domain critical for optimal zinc transport. Nat Commun 7, 11979

20. Hu, J., Wille, H., and Schmitt-Ulms, G. (2018) The Evolutionary unZIPping of a Dimerization Motif-A Comparison of ZIP and PrP Architectures. Pathogens 7, 4

21. Schmitt, S., Kury, S., Giraud, M., Dreno, B., Kharfi, M., and Bezieau, S. (2009) An update on mutations of the SLC39A4 gene in acrodermatitis enteropathica. Hum Mutat 30, 926933

22. Kury, S., Kharfi, M., Schmitt, S., and Bezieau, S. (2012) Clinical utility gene card for: acrodermatitis enteropathica. Eur J Hum Genet 20

23. Hoch, E., Levy, M., Hershfinkel, M., and Sekler, I. (2020) Elucidating the $H(+)$ Coupled $\mathrm{Zn}(2+)$ Transport Mechanism of ZIP4; Implications in Acrodermatitis Enteropathica. Int J Mol Sci 21

24. Waterhouse, A., Bertoni, M., Bienert, S., Studer, G., Tauriello, G., Gumienny, R., Heer, F. T., de Beer, T. A. P., Rempfer, C., Bordoli, L., Lepore, R., and Schwede, T. (2018) SWISSMODEL: homology modelling of protein structures and complexes. Nucleic Acids Res 46, W296-W303

25. Kury, S., Kharfi, M., Kamoun, R., Taieb, A., Mallet, E., Baudon, J. J., Glastre, C., Michel, B., Sebag, F., Brooks, D., Schuster, V., Scoul, C., Dreno, B., Bezieau, S., and Moisan, J. P. (2003) Mutation spectrum of human SLC39A4 in a panel of patients with acrodermatitis enteropathica. Hum Mutat 22, 337-338

26. Nakano, A., Nakano, H., Nomura, K., Toyomaki, Y., and Hanada, K. (2003) Novel SLC39A4 mutations in acrodermatitis enteropathica. J Invest Dermatol 120, 963-966

27. Jung, A. G., Mathony, U. A., Behre, B., Kury, S., Schmitt, S., Zouboulis, C. C., and Lippert, U. (2011) Acrodermatitis enteropathica: an uncommon differential diagnosis in childhood - first description of a new sequence variant. J Dtsch Dermatol Ges 9, 999-1002

28. Zhang, T., Kuliyev, E., Sui, D., and Hu, J. (2019) The histidine-rich loop in the extracellular domain of ZIP4 binds zinc and plays a role in zinc transport. Biochem J 476, 1791-1803

29. Zhang, T., Sui, D., Zhang, C., Cole, L., and Hu, J. (2020) Asymmetric functions of a binuclear metal center within the transport pathway of a human zinc transporter ZIP4. FASEB J 34, 237-247

30. Spiro, R. G. (2002) Protein glycosylation: nature, distribution, enzymatic formation, and disease implications of glycopeptide bonds. Glycobiology 12, 43R-56R

31. Marinko, J. T., Huang, H., Penn, W. D., Capra, J. A., Schlebach, J. P., and Sanders, C. R. (2019) Folding and Misfolding of Human Membrane Proteins in Health and Disease: From Single Molecules to Cellular Proteostasis. Chem Rev 119, 5537-5606

32. Louis-Jeune, C., Andrade-Navarro, M. A., and Perez-Iratxeta, C. (2012) Prediction of protein secondary structure from circular dichroism using theoretically derived spectra. Proteins 80, 374-381

33. Zhou, N. E., Kay, C. M., and Hodges, R. S. (1992) Synthetic model proteins. Positional effects of interchain hydrophobic interactions on stability of two-stranded alpha-helical coiled-coils. J Biol Chem 267, 2664-2670 
34. Legardinier, S., Raguenes-Nicol, C., Tascon, C., Rocher, C., Hardy, S., Hubert, J. F., and Le Rumeur, E. (2009) Mapping of the lipid-binding and stability properties of the central rod domain of human dystrophin. J Mol Biol 389, 546-558

35. Zhang, T., Liu, J., Fellner, M., Zhang, C., Sui, D., and Hu, J. (2017) Crystal structures of a ZIP zinc transporter reveal a binuclear metal center in the transport pathway. Sci Adv 3, e1700344

36. Cheng, S. H., Gregory, R. J., Marshall, J., Paul, S., Souza, D. W., White, G. A., O'Riordan, C. R., and Smith, A. E. (1990) Defective intracellular transport and processing of CFTR is the molecular basis of most cystic fibrosis. Cell 63, 827-834

37. Lukacs, G. L., and Verkman, A. S. (2012) CFTR: folding, misfolding and correcting the DeltaF508 conformational defect. Trends Mol Med 18, 81-91

38. Liu, F., Zhang, Z., Csanady, L., Gadsby, D. C., and Chen, J. (2017) Molecular Structure of the Human CFTR Ion Channel. Cell 169, 85-95 e88

39. Liu, F., Zhang, Z., Levit, A., Levring, J., Touhara, K. K., Shoichet, B. K., and Chen, J. (2019) Structural identification of a hotspot on CFTR for potentiation. Science 364, 1184-1188

40. Gaither, L. A., and Eide, D. J. (2001) The human ZIP1 transporter mediates zinc uptake in human K562 erythroleukemia cells. J Biol Chem 276, 22258-22264

41. Chowanadisai, W., Graham, D. M., Keen, C. L., Rucker, R. B., and Messerli, M. A. (2013) Neurulation and neurite extension require the zinc transporter ZIP12 (slc39a12). Proc NatI Acad Sci U S A 110, 9903-9908

42. Jeong, J., Walker, J. M., Wang, F., Park, J. G., Palmer, A. E., Giunta, C., Rohrbach, M., Steinmann, B., and Eide, D. J. (2012) Promotion of vesicular zinc efflux by ZIP13 and its implications for spondylocheiro dysplastic Ehlers-Danlos syndrome. Proc Natl Acad Sci U S A 109, E3530-3538

43. Mao, X., Kim, B. E., Wang, F., Eide, D. J., and Petris, M. J. (2007) A histidine-rich cluster mediates the ubiquitination and degradation of the human zinc transporter, hZIP4, and protects against zinc cytotoxicity. J Biol Chem 282, 6992-7000

44. Pinilla-Tenas, J. J., Sparkman, B. K., Shawki, A., Illing, A. C., Mitchell, C. J., Zhao, N., Liuzzi, J. P., Cousins, R. J., Knutson, M. D., and Mackenzie, B. (2011) Zip14 is a complex broadscope metal-ion transporter whose functional properties support roles in the cellular uptake of zinc and nontransferrin-bound iron. Am J Physiol Cell Physiol 301, C862-871

45. Bin, B. H., Hojyo, S., Hosaka, T., Bhin, J., Kano, H., Miyai, T., Ikeda, M., Kimura-Someya, T., Shirouzu, M., Cho, E. G., Fukue, K., Kambe, T., Ohashi, W., Kim, K. H., Seo, J., Choi, D. H., Nam, Y. J., Hwang, D., Fukunaka, A., Fujitani, Y., Yokoyama, S., Superti-Furga, A., Ikegawa, S., Lee, T. R., and Fukada, T. (2014) Molecular pathogenesis of spondylocheirodysplastic Ehlers-Danlos syndrome caused by mutant ZIP13 proteins. EMBO Mol Med 6, 1028-1042

46. Fukada, T., Civic, N., Furuichi, T., Shimoda, S., Mishima, K., Higashiyama, H., Idaira, Y., Asada, Y., Kitamura, H., Yamasaki, S., Hojyo, S., Nakayama, M., Ohara, O., Koseki, H., Dos Santos, H. G., Bonafe, L., Ha-Vinh, R., Zankl, A., Unger, S., Kraenzlin, M. E., Beckmann, J. S., Saito, I., Rivolta, C., Ikegawa, S., Superti-Furga, A., and Hirano, T. (2008) The zinc transporter SLC39A13/ZIP13 is required for connective tissue development; its involvement in BMP/TGF-beta signaling pathways. PLoS One 3, e3642

47. Park, J. H., Hogrebe, M., Gruneberg, M., DuChesne, I., von der Heiden, A. L., Reunert, J., Schlingmann, K. P., Boycott, K. M., Beaulieu, C. L., Mhanni, A. A., Innes, A. M., Hortnagel, 
K., Biskup, S., Gleixner, E. M., Kurlemann, G., Fiedler, B., Omran, H., Rutsch, F., Wada, Y., Tsiakas, K., Santer, R., Nebert, D. W., Rust, S., and Marquardt, T. (2015) SLC39A8 Deficiency: A Disorder of Manganese Transport and Glycosylation. Am J Hum Genet 97, 894-903

48. Boycott, K. M., Beaulieu, C. L., Kernohan, K. D., Gebril, O. H., Mhanni, A., Chudley, A. E., Redl, D., Qin, W., Hampson, S., Kury, S., Tetreault, M., Puffenberger, E. G., Scott, J. N., Bezieau, S., Reis, A., Uebe, S., Schumacher, J., Hegele, R. A., McLeod, D. R., Galvez-Peralta, M., Majewski, J., Ramaekers, V. T., Care4Rare Canada, C., Nebert, D. W., Innes, A. M., Parboosingh, J. S., and Abou Jamra, R. (2015) Autosomal-Recessive Intellectual Disability with Cerebellar Atrophy Syndrome Caused by Mutation of the Manganese and Zinc Transporter Gene SLC39A8. Am J Hum Genet 97, 886-893

49. Choi, E. K., Nguyen, T. T., Gupta, N., Iwase, S., and Seo, Y. A. (2018) Functional analysis of SLC39A8 mutations and their implications for manganese deficiency and mitochondrial disorders. Sci Rep 8, 3163

50. Taylor-Cousar, J. L., Munck, A., McKone, E. F., van der Ent, C. K., Moeller, A., Simard, C., Wang, L. T., Ingenito, E. P., McKee, C., Lu, Y., Lekstrom-Himes, J., and Elborn, J. S. (2017) Tezacaftor-Ivacaftor in Patients with Cystic Fibrosis Homozygous for Phe508del. N Engl J Med 377, 2013-2023

51. Hoogenraad, T. U., Dekker, A. W., and van den Hamer, C. J. (1985) Copper responsive anemia, induced by oral zinc therapy in a patient with acrodermatitis enteropathica. Sci Total Environ 42, 37-43

52. Fischer, P. W., Giroux, A., and L'Abbe, M. R. (1984) Effect of zinc supplementation on copper status in adult man. Am J Clin Nutr 40, 743-746 


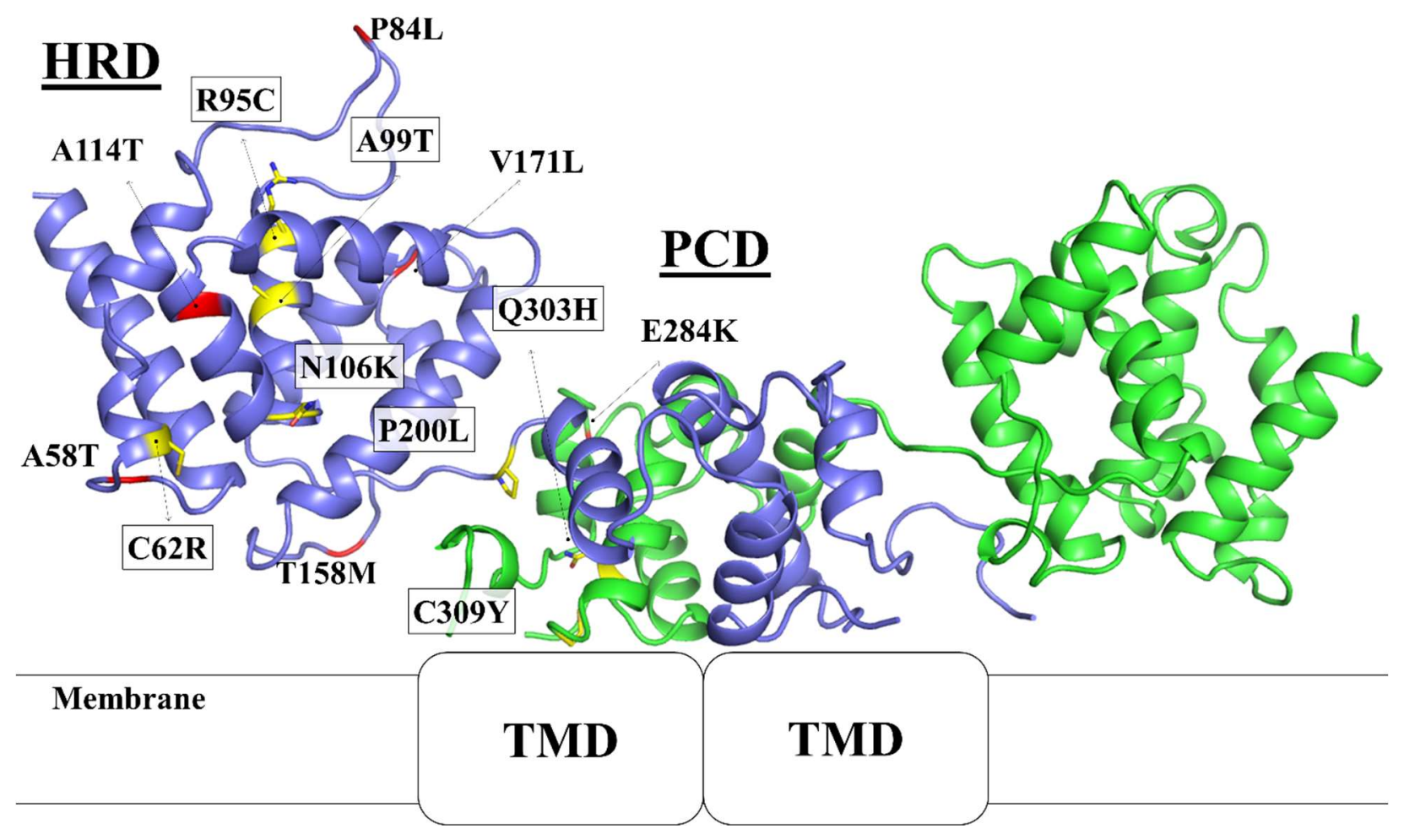

Figure 1. Mapping of the AE-causing mutations and the SNPs on the structural model of hZIP4ECD dimer. Seven residues subjected to AE-causing mutations (highlighted in boxes) are in yellow and stick mode with four in the HRD domain and three in the PCD domain, whereas the residues at which SNPs occur are in red. Additional 3 SNPs not shown in the model are either in the highly disordered his-rich loop (R251W) or in the signal peptide (V2A and E10A). 

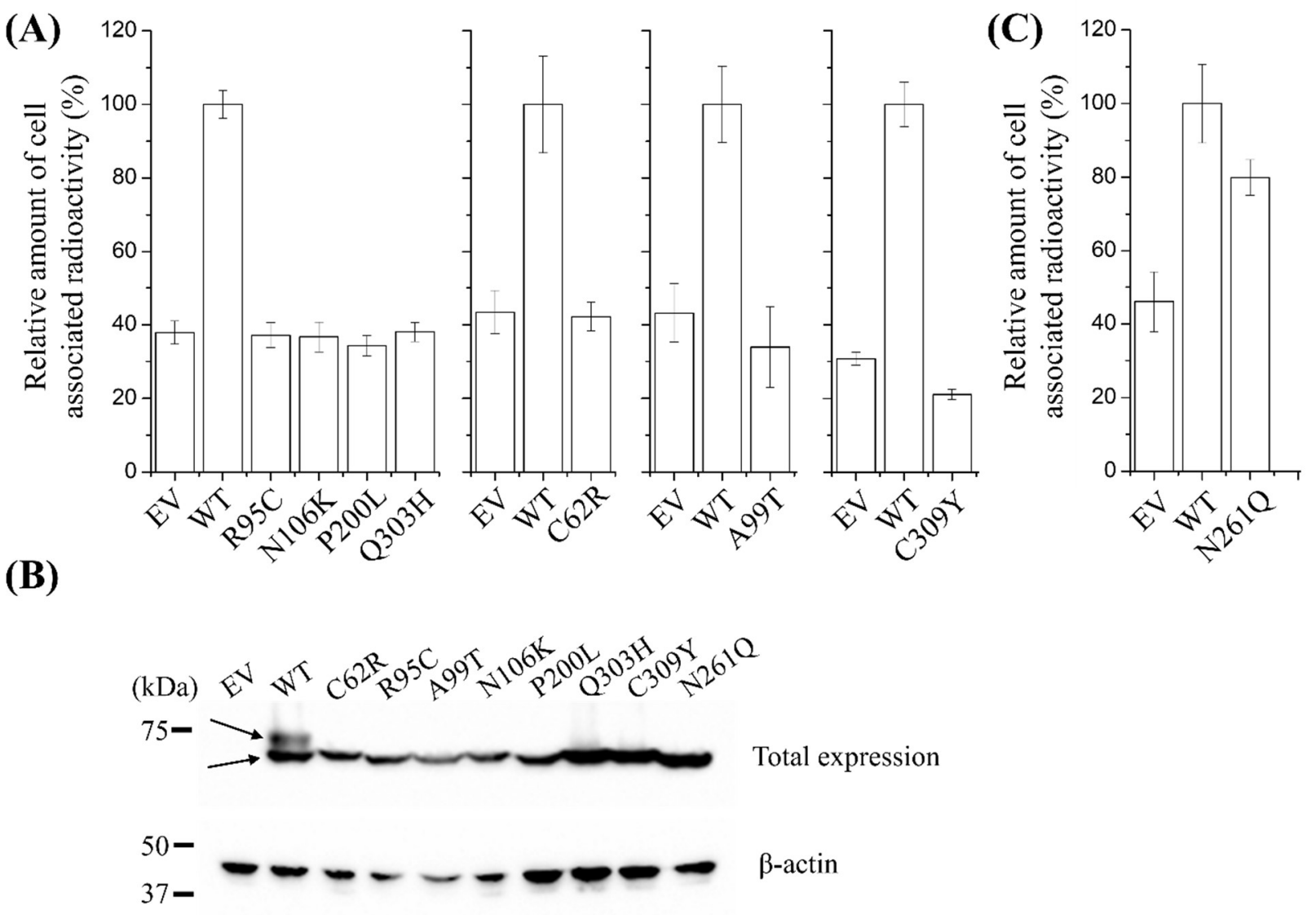

Figure 2. Functional characterization of hZIP4 and the variants. (A) Cell-based zinc transport assay of the $\mathrm{AE}$-associated variants with $10 \mu \mathrm{M} \mathrm{Zn}^{2+}$ in the medium. The results of one out of 26 independent experiments are shown. Three technical repeats were included in each experiment. The error bars indicate \pm 1 standard deviation. (B) Total expression hZIP4 and the variants detected by Western blot using anti-HA antibody. The upper arrow indicates the maturely glycosylated hZIP4, whereas the lower arrow indicates the immaturely glycosylated form. $\beta$-actin was detected in the same sample as loading control. (C) Zinc transport assay of the N261Q variant. Three technical repeats were included in the experiment. The error bars indicate \pm 1 standard deviation. 
(A)

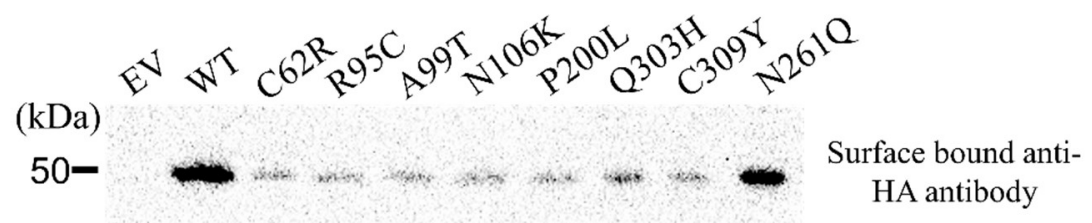

$75-$ HA antibody

(B)

Non-permeabilized cells

(surface expression)

Permeabilized cells (total expression)

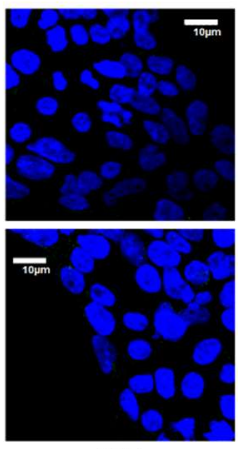

EV

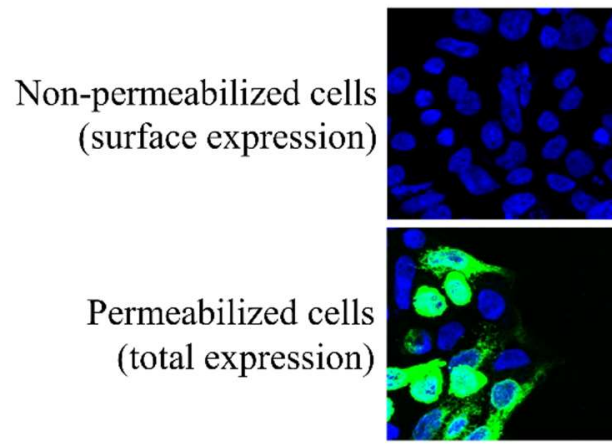

N106K

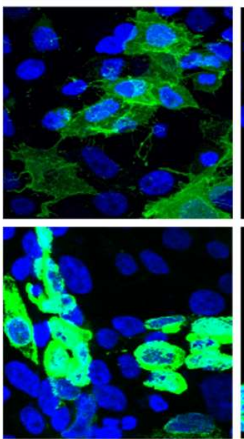

WT

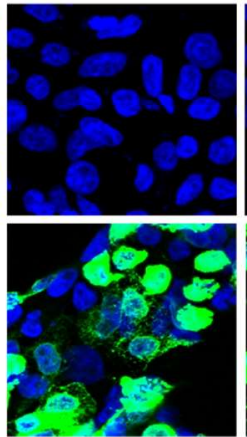

P200L

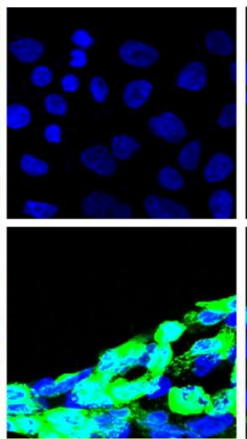

C62R

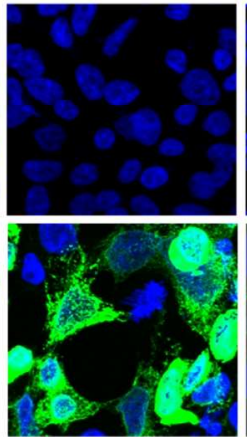

Q303H

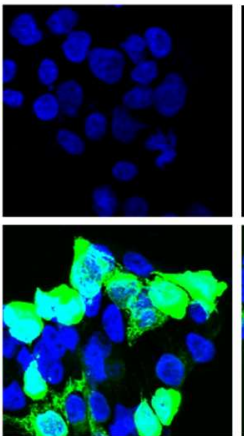

R95C

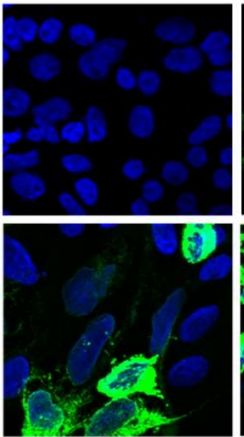

C309Y

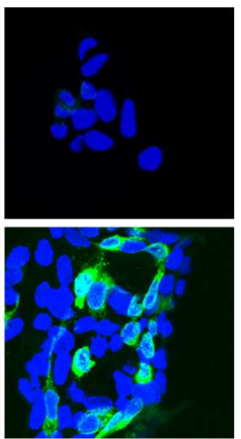

A99T

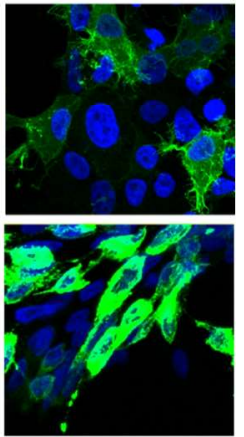

N261Q

Figure 3. Cell surface expression of hZIP4 and its variants. (A) Surface expression detected by Anti-HA antibody in Western blot. Total expression of hZIP4 and the variants from the same batch of cells are shown for comparison. $\beta$-actin in the same sample was detected as loading control. (B) Detection of expression of hZIP4 and its variants. FITC-labeled anti-HA antibody was used to stain HA tagged hZIP4 in non-permeabilized cells (upper row, for detection of cell surface expression) or permeabilized cells (lower row, for detection of total expression in whole cells). The scale bar $=10 \mu \mathrm{m}$. 


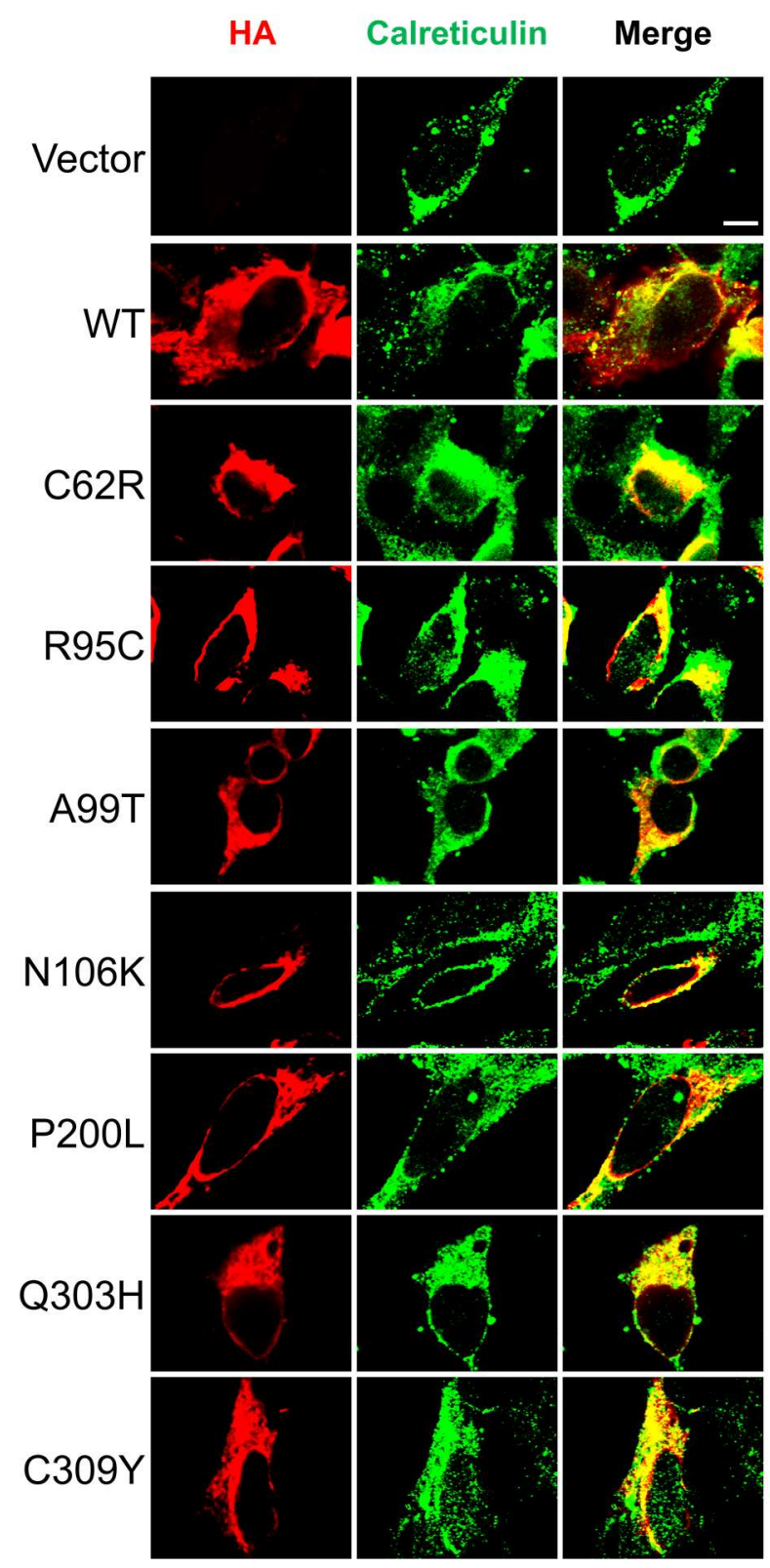

Figure 4. Colocalization of hZIP4 with ER marker calreticulin. Representative confocal images (100X) of HEK293T cells transiently expressing hZIP4-HA or the variants are shown. hZIP4 and its variants were detected with an anti-HA tag antibody and an Alexa-568 goat anti-mouse antibody (red). Calreticulin was detected with an anti-calreticulin antibody and an Alexa-488 antirabbit antibody (green). Scale bar $=5 \mu \mathrm{m}$. 

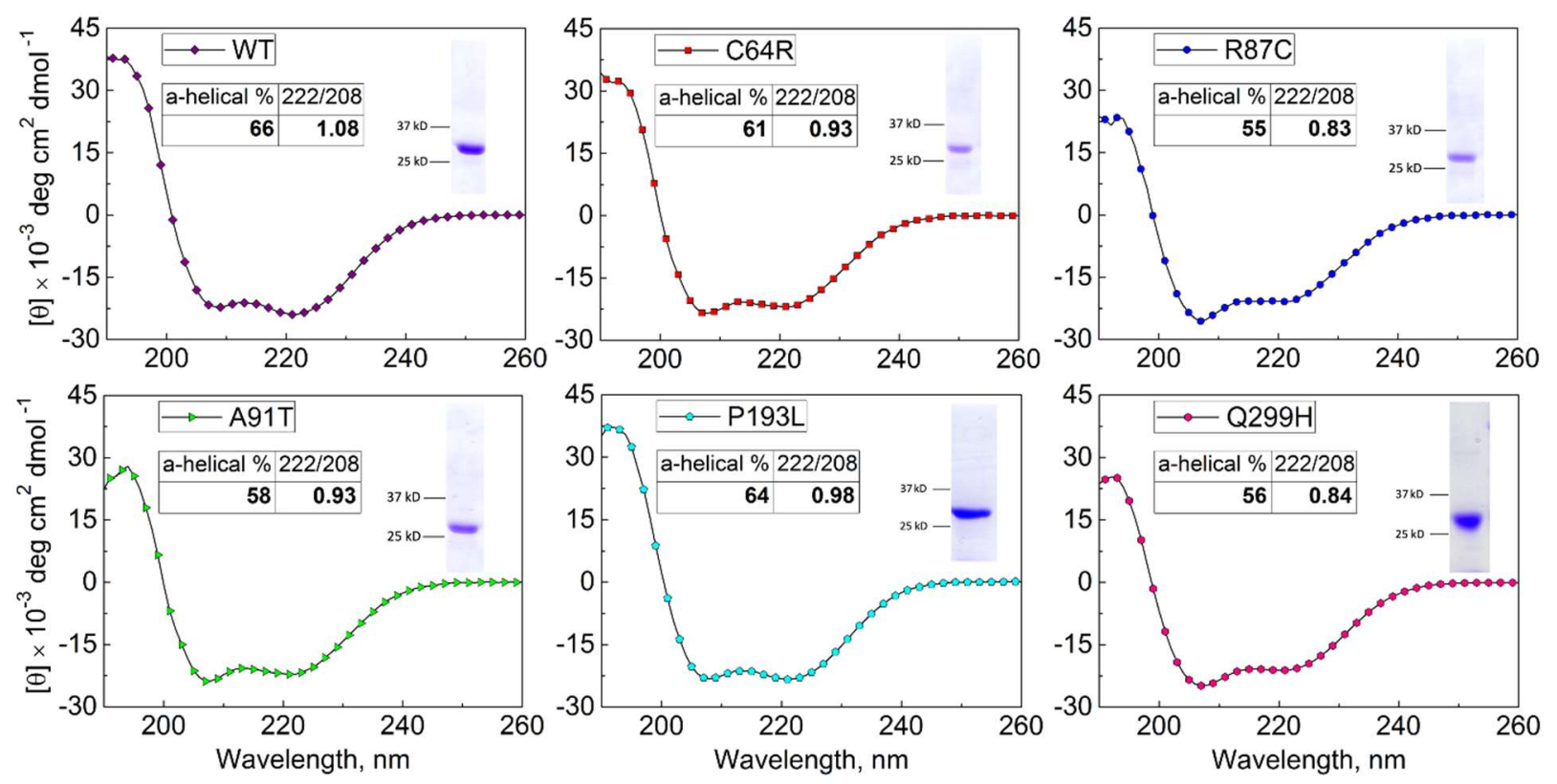

Figure 5. CD spectra of pZIP4-ECD and the variants. The $\alpha$-helical content was estimated using the K2D3 server. 222/208 indicates the ratio of $\theta_{222} / \theta_{208}$. The SDS-PAGEs of purified protein are shown in the insets. 


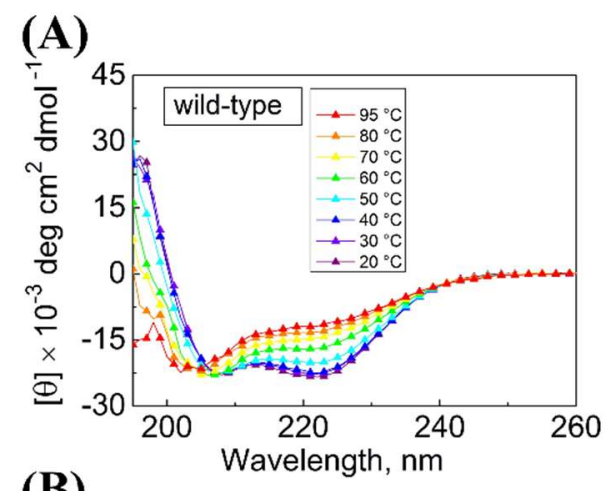

(B)
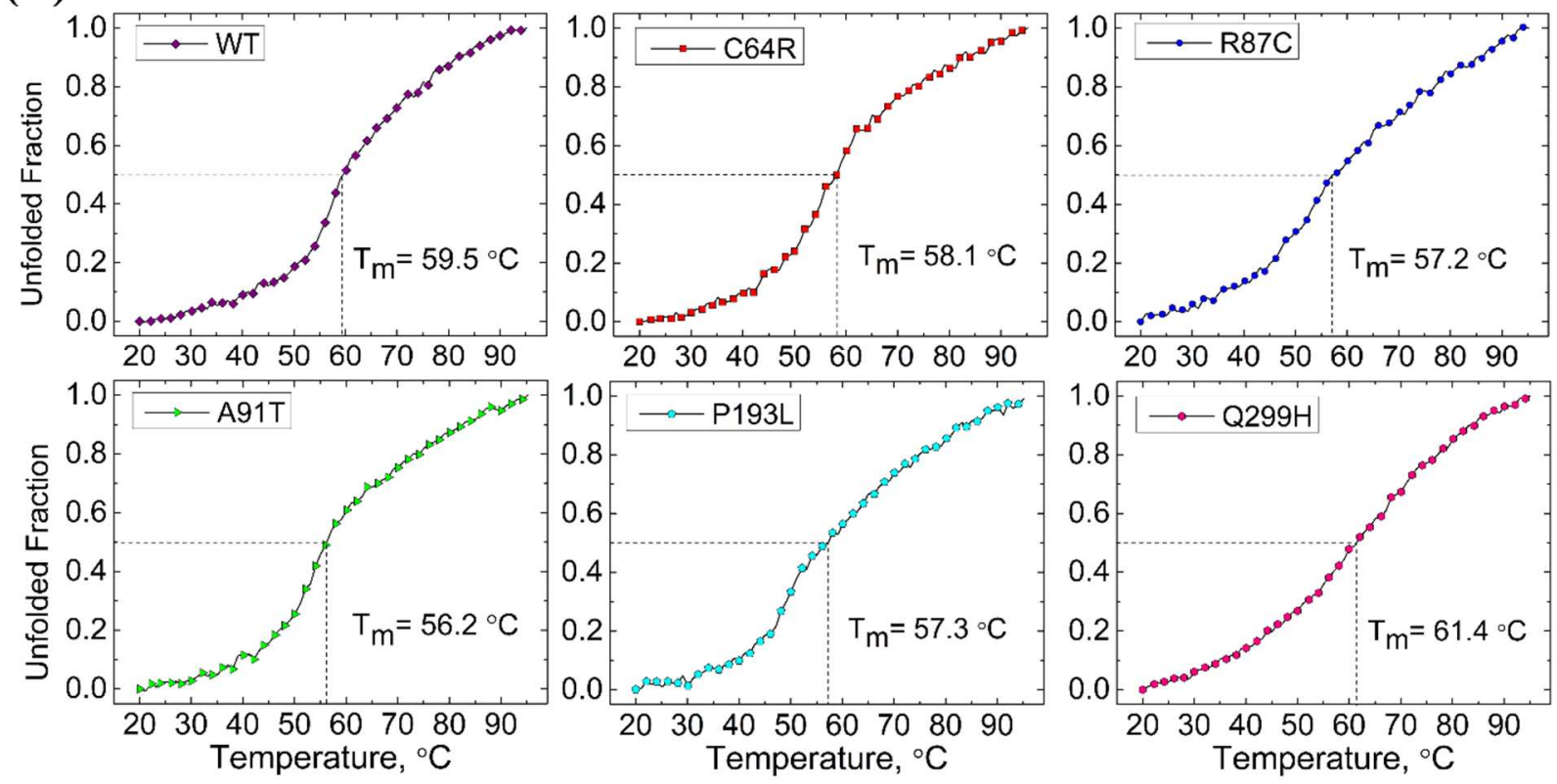

Figure 6. Heat denaturation of pZIP4-ECD and the variants. (A) CD spectra of wild type pZIP4ECD at different temperatures. At $95{ }^{\circ} \mathrm{C}$, the $\alpha$-helical content was estimated to be $20 \%$ and further increasing temperature did not reduce helical content. (B) Heat denaturation curves of the wild type pZIP4-ECD and the variants. The melting temperature (Tm) was estimated on the denaturation curve where the unfolded fraction is 0.5 . 


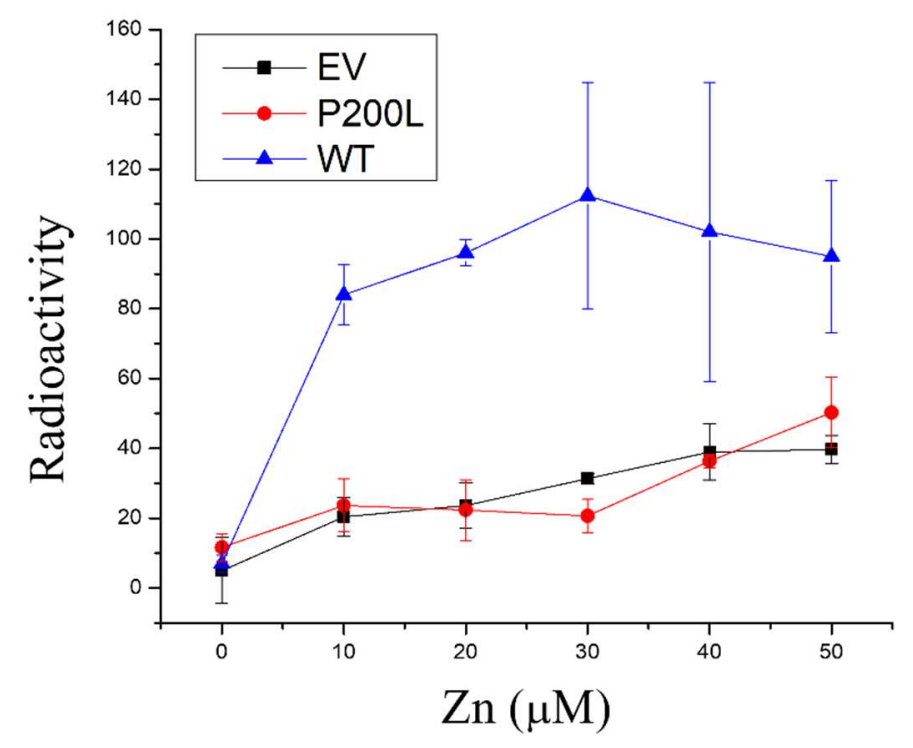

Figure S1. Zinc transport activity measurement at indicated zinc concentrations. Three technical repeats were averaged at each data point. The error bars indicate $1 \pm$ standard deviation. 


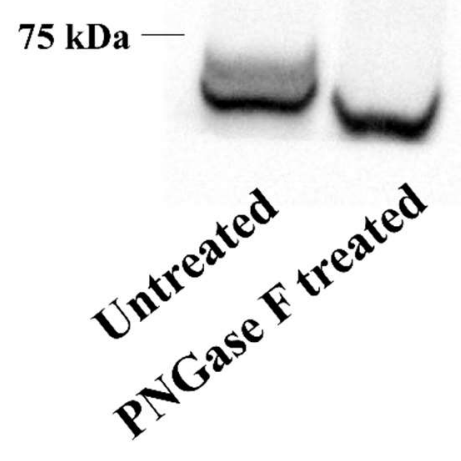

Figure S2. Processing of the wild type hZIP4 by PNGase F. PNGase F was directly added to the SDS-PAGE sample. 

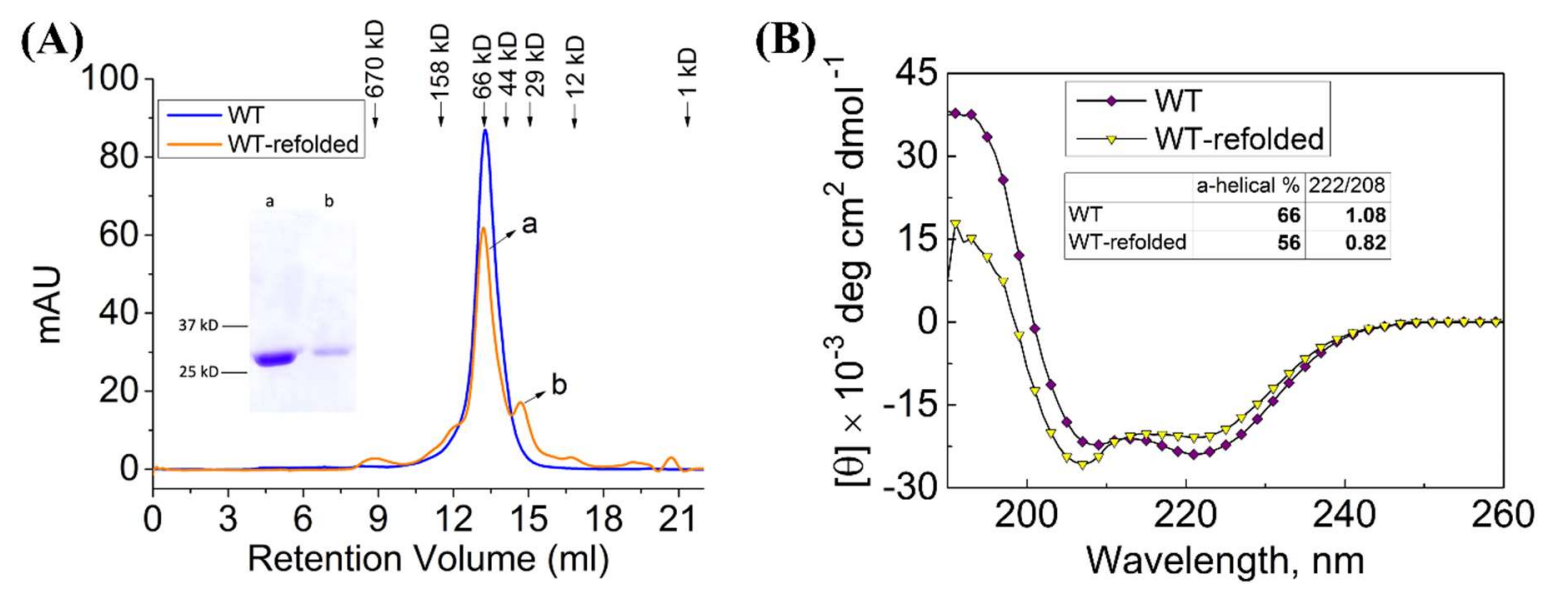

Figure S3. Characterization of refolded wild type pZIP4-ECD. (A) Size exclusion chromatography of the native and the refolded proteins. The elution volumes of the standard proteins are indicated by arrows. Note that the refolded protein has a major peak (peak a, dimer) and a minor peak (peak $b$, monomer). (B) CD spectra of the native and the refolded proteins. The refolded protein has lower $\alpha$-helical content and smaller $\theta_{222} / \theta_{208}$ ratio than the wild type protein. 